\title{
PRODUCTION GAMES, CORE DEFICIT, DUALITY AND SHADOW PRICES
}

\author{
SJUR DIDRIK FLÅM \\ Department of Economics, University of Bergen \\ Hermann Foss gt. 6, 5007 Bergen, Norway \\ E-mail: sjur.flaam@econ.uib.no
}

\begin{abstract}
Considered here are production (or market) games with transferable utility. Prime objects are explicitly computable core solutions, or somewhat "deficit" versions of such, fully defined by shadow prices. Main arguments revolve around standard Lagrangian duality. A chief concern is to relax, or avoid, the commonplace assumption that all preferences and production possibilities be convex. Doing so, novel results are obtained about non-emptiness of the core, and about specific imputations therein.
\end{abstract}

1. Introduction. Considered below are cooperative, transferable-utility $(T U)$ games among parties concerned with equitable sharing of efficient production costs. There is a nonempty finite player set $I$, and coalition $S \subseteq I$ would incur cost $C_{S} \in \mathbb{R} \cup\{+\infty\}$ if going alone. ${ }^{1}$ Reasonably assume, as a weak form of subadditivity, that

$$
C_{I} \leq \sum_{i \in I} C_{i}<+\infty
$$

Denote by $\mathbb{C}$ the characteristic function $S \mapsto C_{S}$. A cost profile $c=\left(c_{i}\right) \in \mathbb{R}^{I}$ is then declared in the core - as signaled by writing $c \in$ core $(\mathbb{C})$, or more simply $c \in$ core -iff it embodies

(I) full cost cover: $\quad \sum_{i \in I} c_{i} \geq C_{I}$ and

(II) coalitional stability: $\sum_{i \in S} c_{i} \leq C_{S}$ for each subset $S \subseteq I$. $\}$

Plainly, this solution concept makes good sense when core is neither empty, nor too large,

2000 Mathematics Subject Classification: 90C26, 90C47, 91A12.

JEL Classification: C71.

Key words and phrases: production games, core, deficit, duality gap.

Support from INDAM, Ruhrgas and Finansmarketsfondet is gratefully acknowledged.

The paper is in final form and no version of it will be published elsewhere.

${ }^{1}$ For the empty coalition set $C_{\emptyset}=0$, and posit each sum $\sum_{\emptyset}=0$. 
nor very sensitive to data. Sensitivity can be mitigated by replacing each "participation constraint" (II) with the corresponding relaxed form: $\sum_{i \in S} c_{i} \leq C_{S}+\varepsilon|S|, \varepsilon>0$. Such relaxation, while yielding so-called $\varepsilon$-approximate cores [12], is quite convenient, but not always most natural. ${ }^{2}$ In fact, regarding possible emptiness of the core, note that coalitional stability - or voluntary participation - obtains easily by charging each player too little payment. So, the real bite resides in the requirement that the overall charge $\sum_{i \in I} c_{i}$ covers the total cost $C_{I}$.

This simple observation indicates that valuable insights might sometimes be gained by relaxing merely inequality (I) to read instead:

$$
\sum_{i \in I} c_{i} \geq C_{I}-D
$$

Here $D \geq 0$ stands for a monetary deficit. Or, $D$ is a debt the grand coalition $I$ contracts with outside creditors. Sometimes, if $I$ can get away without paying $C_{I}$ in full, $D$ might be construed as a damage inflicted on the environment. A quite parallel story concerns of course profit sharing. All inequalities in (2) are then reversed, and (3) assumes the form $\sum_{i \in I} c_{i} \geq C_{I}+D$, featuring a "donation" $D \geq 0$. That twin story will not be told here. To begin and stay with cost sharing, consider rather

EXAMPLE 1.1. Accommodated right here are three producers, each obliged to put out precisely the amount $e_{i}=1 / 2$ of the same homogenous commodity. Cost functions $f_{i}$ : $\mathbb{X}_{i} \rightarrow \mathbb{R}$ are equal, and defined by $f_{i}\left(x_{i}\right):=$ the smallest integer $\geq x_{i} \in \mathbb{X}_{i}=[0,1]$. Coalition $S$ incurs cost

$$
C_{S}:=\inf \left\{\sum_{i \in S} f_{i}\left(x_{i}\right): \sum_{i \in S} x_{i}=\sum_{i \in S} e_{i}\right\} .
$$

Clearly, $C_{S}=1$ when $|S| \leq 2$. If some $\left(c_{i}\right) \in$ core, then $c_{i}+c_{j} \leq 1$ for every twoplayer coalition $S=\{i, j\}$. Summation of all these two-player inequalities produces the contradiction $\sum_{i \in I} c_{i} \leq 3 / 2<C_{I}=2$. Thus, because $C_{I}$ is "too large", core is empty. A substitution $C_{I} \leftarrow C_{I}-d$, using minimal deficit $d=1 / 2$, suffices though to have $\left(\frac{1}{2}, \frac{1}{2}, \frac{1}{2}\right)$ as the single core element. Alternatively, replacing $f_{i}$ with the largest convex function $\check{f}_{i} \leq f_{i}$ in (4), we get $\check{f}_{i}\left(x_{i}\right)=x_{i}$, and modified coalition costs $\check{C}_{S}=|S| / 2$, these data again admitting $\left(\frac{1}{2}, \frac{1}{2}, \frac{1}{2}\right)$ as unique core element. However, upon letting $\mathbb{X}_{i}=\{0,1\}$ with $f_{i}\left(x_{i}\right)=+\infty$ whenever $x_{i} \notin \mathbb{X}_{i}$, coalition cost equals 1 if $|S|=2,+\infty$ otherwise, and again core $=\varnothing$.

The temptation - or need - to tolerate a deficit $D$ in (I) makes it natural to declare $c=\left(c_{i}\right) \in \mathbb{R}^{I}$ in the $D$-core $(\mathbb{C})$-and write simply $c \in D$-core-iff it satisfies (3) and $\sum_{i \in S} c_{i} \leq C_{S} \forall S \subseteq I$. The set so defined is a polyhedron (i.e. an intersection of closed half-spaces). Further, (1) implies $C_{I}-D-\sum_{j \neq i} C_{j} \leq c_{i} \leq C_{i}$, so $D$-core must be bounded - whence a polytope (i.e. the convex hull of finitely many points, if any). Since $D$-core decreases with $D$, the smallest possible deficit is worth closer scrutiny.

\footnotetext{
${ }^{2}$ In particular, why should the perturbation be "uniform" across coalitions? And how does $\varepsilon$ relate to underlying data?
} 
Proposition 1.1 (On the minimal deficit). Let $d:=\inf \{D \geq 0: D$-core $\neq \emptyset\}$ denote the minimal deficit. Then $d$-core is nonempty, and

$$
d \text {-core }=\cap\{D \text {-core }: D>d\} .
$$

Moreover, in terms of a primal-dual pair of linear programs, we have

$$
\begin{gathered}
C_{I}-d=\max \left\{\sum_{i \in I} c_{i}: \sum_{i \in S} c_{i} \leq C_{S} \text { for all } S \subseteq I\right\} \\
=\min \left\{\sum_{S} w_{S} C_{S}: w_{S} \geq 0 \text { for all } S \subseteq I, \text { and } \sum_{S: i \in S} w_{S}=1 \text { for all } i \in I\right\} .
\end{gathered}
$$

Thus, $D$-core $(\mathbb{C})=\operatorname{core}\left(\mathbb{C}-D \mathbf{1}_{I}\right)$ for all $D \geq 0$, and nonempty iff $D \geq d .{ }^{3}$

The proof of Proposition 1.1 follows straightforwardly from standard linear programming duality: Plainly, (Primal) and (Dual) are both feasible. Therefore, their optimal values are attained and equal. Immediately one recovers here a well known result:

Corollary 1.1 (Bondareva [2], Shapley [15]). Deficit $d=0 \Leftrightarrow$ core is nonempty $\Leftrightarrow$ the game is balanced $\Longleftrightarrow C_{I} \leq \sum_{S} w_{S} C_{S}$ for all families $\left(w_{S}\right) \geq 0$ of weights, balanced in that $\sum_{S: i \in S} w_{S}=1 \forall i$.

Proposition 1.1 might lure one into thinking that, in principle, no more need be said. This is certainly not so. At least four queries come straight up:

First, either linear program (Primal) and (Dual) presumes explicit knowledge of all $\operatorname{costs} C_{S}$, in total $2^{|I|}-1$ of them. Typically, to generate these numbers is quite a task. So, an easier approach would be welcome. In addition, "solution" of a game typically involves somewhat more detail than mere satisfaction of a large, linear inequality system.

Second, it appears worthwhile to assess, or estimate, $d$ in terms of primitive data, if any, underlying the characteristic function $S \mapsto C_{S}$. Instead of first generating that function, and study it thereafter, one might want to leave structure pretty much as is and explore the game in original form.

Third - for the sake of computation, interpretation or implementation - it is desirable to display explicit core solutions, or approximate versions of such, in terms of nonprocessed data.

Fourth and finally, if $d>0$, that is, if the game features some "imbalance", one would like to see exactly where and why.

This paper offers a vista on these issues as they relate to production and market games. Admittedly, such games are somewhat special, but of frequent occurrence and

${ }^{3}$ To mitigate instances having $d>0$ and empty core, when $C_{I} /\left(C_{I}-d\right)>0$, one may follow Gomez [7] in defining a core extension by

$$
\operatorname{core} \operatorname{Ext}(\mathbb{C}):=\frac{C_{I}}{C_{I}-d} \operatorname{core}\left(\mathbb{C}-d \mathbf{1}_{I}\right) .
$$

Also, upon replacing (Primal) by the more constrained program

$$
\max \sum_{i \in I} c_{i} \text { s.t. (II) and each } i \in \text { some } S(i) \subseteq I \text { such that } \sum_{s \in S(i)} c_{s} \geq C_{S(i)},
$$

one arrives at what is called the aspiration core, studied in [3], [8], [11]. 
great importance. A sample of studies include [5], [6], [9], [10], [13], [14], [17] and references therein.

The objectives and novelties of the paper are twofold: First, after recalling that the minimal deficit relates to the much studied duality gap in optimization, we use related theory to provide a new condition, concerning weak stability, which entails $d=0$ whence core $\neq \emptyset$. Second, provided the production game be stable, we shall exhibit explicit core solutions, generated by so-called shadow prices. Numerous examples are included.

Motivation for this inquiry stems from the need to reach beyond instances with convex preferences and production sets. Notably, there should be ample room for discrete activity (decision) sets, non-divisibility of various inputs, and non-transferability of specific production factors. What imports though, is that some key resources (or production tasks) be perfectly divisible and transferable (maybe modulo time-shared use). Bundles of these resources are privately owned. Their scarcity generates common willingness to pay for appropriation or utilization. Thus emerge endogenous shadow prices or user costs. In their turn these determine specific core imputations - albeit only up to deficit $D \geq d$.

Section 2 defines the games and includes a running example. Section 3 reviews some non-standard, novel results on Lagrangian duality that prove helpful. Section 4 contains the main results, believed to interest several sorts of readers. Particularly addressed are mathematicians, more concerned with optimization than with games - and economists, looking more at markets than at duality.

The following notation is convenient. When $C$ is a (constraint) subset of some ambient space, denote by $\delta_{C}$ the extreme penalty (or indicator) function that equals 0 on $C$ and $+\infty$ elsewhere. Whenever $y(\cdot)$ is a linear, real-valued function, write $\langle y, e\rangle$ instead of $y(e)$.

2. Production games. In the present setting, a nonempty coalition $S$, if it were to form, would attempt to incur minimal cost

$$
C_{S}:=\inf \left\{f_{S}\left(x_{S}\right)+h_{S}\left(g_{S}\left(x_{S}\right)\right)\right\} .
$$

Here the function $f_{S}$ takes a prescribed, but abstract set $\mathbb{X}_{S}$ into $\mathbb{R} \cup\{+\infty\}$; the operator $g_{S}$ maps $\mathbb{X}_{S}$ into a real vector space $\mathbb{E}$; and finally, $h_{S}: \mathbb{E} \rightarrow \mathbb{R} \cup\{+\infty\}$ is a sort of penalty function. At this juncture, no topological or geometric properties are demanded of $\mathbb{X}_{S}, \mathbb{E}, f_{S}, g_{S}, h_{S}$.

For interpretation construe $f_{S}: \mathbb{X}_{S} \rightarrow \mathbb{R} \cup\{+\infty\}$ as an aggregate cost function, $x_{S} \in \mathbb{X}_{S}$ denoting the activity, production plan, or technological design that coalition $S$ opts for. Further, let $g_{S}$ govern - and account for-resource consumption or technological restrictions. Finally, $h_{S}: \mathbb{E} \rightarrow \mathbb{R} \cup\{+\infty\}$ should be seen as a penalty mechanism meant to enforce feasibility. Note that $\mathbb{E}$, the vector space of "resource endowments" or "production commitments", is common to all agents and coalitions. By tacit assumption $h_{S}\left(g_{S}\left(x_{S}\right)\right)=+\infty$ when $x_{S} \notin \mathbb{X}_{S}$, and the convention $+\infty-\infty=+\infty$ always applies.

To our knowledge $T U$ production (or market) games have hitherto not been defined in such generality. Format $\left(P_{S}\right)$ can accommodate a wide variety of instances. To wit, consider

EXAMPLE 2.1 (Nonlinear constrained, cooperative programming). For each $i \in I$ there is a nonempty set $\mathbb{X}_{i}$, two functions $f_{i}: \mathbb{X}_{i} \rightarrow \mathbb{R} \cup\{+\infty\}, g_{i}: \mathbb{X}_{i} \rightarrow \mathbb{E}$, and a set constraint 
$g_{i}\left(x_{i}\right) \in K_{i} \subset \mathbb{E}$. Let then $\mathbb{X}_{S}:=\Pi_{i \in S} \mathbb{X}_{i}$. Further, posit $f_{S}\left(x_{S}\right):=\sum_{i \in S} f_{i}\left(x_{i}\right)$ and $g_{S}\left(x_{S}\right):=\sum_{i \in S} g_{i}\left(x_{i}\right)$. Finally, introduce $K_{S}:=\sum_{i \in S} K_{i}$ and let $h_{S}:=\delta_{K_{S}}$ be the corresponding extreme indicator.

Of particular importance is the special case where $\mathbb{X}_{i}=\mathbb{E}, g_{i}\left(x_{i}\right)=x_{i}-e_{i}$, and $K_{i}=\{0\}$. Coalitional cost $C_{S}$ is then defined by infimal convolution (4).

To advertize the appeal of games in form $\left(P_{S}\right)$ it is natural to state forthwith:

Proposition 2.1 (Convexity and separability ensures nonempty core). Suppose $\mathbb{X}_{S}=$ $\Pi_{i \in S} \mathbb{X}_{i}$ with each $\mathbb{X}_{i}$ convex. Also suppose

$$
f_{S}\left(x_{S}\right)+h_{S}\left(g\left(x_{S}\right)\right) \geq \sum_{i \in S} F_{i}\left(x_{i}\right)+\delta_{\{0\}}\left(\sum_{i \in S} \mathcal{A}_{i} x_{i}\right) \forall S \subseteq I,
$$

with each $F_{i}$ convex, each $\mathcal{A}_{i}: \mathbb{X}_{i} \rightarrow \mathbb{E}$ affine, and with equality for $S=I$. Then the core is nonempty.

Proof. Let $\left(w_{S}\right) \geq 0$ be any balanced collection of weights; that is, $\sum_{S: i \in S} w_{S}=1$ for all $i$. Pick any positive $\varepsilon$ and for each nonempty coalition $S$ a profile $x_{S}=\left(x_{i S}\right) \in \mathbb{X}_{S}$ such that $f_{S}\left(x_{S}\right)+h_{S}\left(g_{S}\left(x_{S}\right)\right) \leq C_{S}+\varepsilon$ and $\sum_{i \in S} \mathcal{A}_{i} x_{i S}=0$. Posit $x_{i}:=\sum_{S: i \in S} w_{S} x_{i S}$. Then $x_{i} \in \mathbb{X}_{i}, \sum_{i \in I} \mathcal{A}_{i} x_{i}=0$, and

$$
C_{I} \leq \sum_{i \in I} F_{i}\left(\sum_{S: i \in S} w_{S} x_{i S}\right) \leq \sum_{i \in I} \sum_{S: i \in S} w_{S} F_{i}\left(x_{i S}\right)=\sum_{S} w_{S} \sum_{i \in S} F_{i}\left(x_{i S}\right) \leq \sum_{S} w_{S}\left[C_{S}+\varepsilon\right] .
$$

Since $\varepsilon>0$ was arbitrary, it follows that $C_{I} \leq \sum_{S} w_{S} C_{S}$. Proposition 1.1 now yields $d=0$.

Proposition 2.1 indicates good prospects for finding nonempty cores. It provides, however, less than full satisfaction. First, no explicit solution is listed. Second, "too much" convexity is presumed in activity sets $\mathbb{X}_{i}$ and cost functions $F_{i}$. Third, resource aggregation is "too linear." And finally, original data somehow disappear. These drawbacks motivate a closer look next at problems of format $\left(P_{S}\right)$, a chief aim being to isolate - or identify - exactly where, and to what extent, convexity is needed.

3. Multipliers and subgradients with deficits. Problem class $\left(P_{S}\right)$ invites a study of the corresponding generic format

$$
\inf \{f(x)+h(g(x))\}
$$

Incorporated here are mappings $f: \mathbb{X} \rightarrow \mathbb{R} \cup\{ \pm \infty\}, g: \mathbb{X} \rightarrow \mathbb{E}$, and $h: \mathbb{E} \rightarrow \mathbb{R} \cup\{ \pm \infty\}$. The set $\mathbb{X}$ is abstract, but $\mathbb{E}$ is a real vector space. By tacit assumption $f(x)+h(g(x))=$ $+\infty$ if $x \notin \mathbb{X}$. Our analysis revolves around the perturbed function

$$
(x, e, y) \in \mathbb{X} \times \mathbb{E} \times \mathbb{Y} \mapsto f(x)+h(g(x)+e)-\langle y, e\rangle .
$$

Here $\mathbb{Y}$ is a judiciously chosen, convex, nonempty set of linear functionals $y: \mathbb{E} \rightarrow \mathbb{R}$. The appropriate nature of $\mathbb{Y}$ is made precise later. This means that additional properties of the functionals $y$ (besides linearity) will be invoked only when needed. As customary, the expression $\langle y, e\rangle$ stands for $y(e)$. Objective (5) features a perturbation $e$ available at a premium $\langle y, e\rangle$. Thus, (5) relaxes and imbeds problem $(P)$ into a competitive market 
where any endowment $e \in \mathbb{E}$ is evaluated at constant "unit price" $y$. To reflect on this situation, associate the Fenchel conjugate

$$
h^{*}(y):=\sup \{\langle y, e\rangle-h(e): e \in \mathbb{E}\}
$$

to $h$. If $h(e)$ denotes the cost of an enterprise that produces output $e$ at revenues $\langle y, e\rangle$, then $h^{*}(y)$ reports the corresponding profit. In economic jargon: the firm in question is a price-taker in the output market. To avoid having $h^{*} \equiv+\infty$, suppose henceforth that $h>-\infty$. Clearly, $h^{*}: \mathbb{Y} \rightarrow \mathbb{R} \cup\{ \pm \infty\}$ is convex. The relaxed objective (5) naturally generates a Lagrangian

$$
\begin{aligned}
L(x, y) & :=\inf \{f(x)+h(g(x)+e)-\langle y, e\rangle: e \in \mathbb{E}\} \\
& =\quad f(x)+\langle y, g(x)\rangle-h^{*}(y),
\end{aligned}
$$

defined on $\mathbb{X} \times \mathbb{Y}$. Call now $y \in \mathbb{Y}$ a $D$-multiplier iff, for given deficit $D \geq 0$, it belongs to the set

$$
M_{D}(P):=M_{D}:=\left\{y \in \mathbb{Y}: \inf _{x} L(x, y) \geq \inf (P)-D\right\} .
$$

Note that $M_{D}$ is convex. Elements in $M:=M_{0}$, if any, are hereafter named Lagrange multipliers. Intimately related to problem $(P)$ is the marginal (optimal value) function

$$
e \in \mathbb{E} \mapsto V(e):=\inf \{f(x)+h(g(x)+e): x \in \mathbb{X}\}
$$

Reasonably assume that its effective domain $\operatorname{dom} V:=V^{-1}(\mathbb{R})$ be nonempty, in which case function $V$ is declared proper. Of prime interest are differential properties of $V(\cdot)$ at the distinguished point $e=0$ :

Definition. Given $D \geq 0$, the functional $y \in \mathbb{Y}$ is called a $D$-subgradient (or subgradient with deficit $D)$ of a proper function $V: \mathbb{E} \rightarrow \mathbb{R} \cup\{+\infty\}$ at $e$, written $y \in \partial_{D} V(e)$, iff

$$
V(\cdot) \geq V(e)+\langle y, \cdot-e\rangle-D .
$$

$V$ is called weakly subdifferentiable at $e$ iff $\partial_{D} V(e)$ is nonempty for each $D>0$, and subdifferentiable at that same point iff $\partial_{0} V(e)=: \partial V(e)$ is nonempty.

Clearly, $y \in \partial_{D} V(e)$ iff $V(e)$ is finite and the affine function $V(e)+\langle y, \cdot-e\rangle$, up to a vertical deficit $D \geq 0$, bounds $V(\cdot)$ globally from below. Note that $\partial_{D} V(e)$ is convex, and it increases with $D$.

Proposition 3.1 (D-supergradient $=D$-multiplier [4]). Suppose $\inf (P)=V(0)$ is finite. Then

$$
\partial_{D} V(0)=M_{D}
$$

Proof. [4] already contains the simple proof, reproduced here for completeness. Letting $\bar{e}:=g(x)+e$ we get

$$
\begin{array}{ll}
y \in \partial_{D} V(0) & \Leftrightarrow \\
f(x)+h(\bar{e})=f(x)+h(g(x)+e) \geq V(e) \geq V(0)+\langle y, e\rangle-D \quad \forall(x, e) \in \mathbb{X} \times \mathbb{E} \Leftrightarrow & \Leftrightarrow \\
f(x)+\langle y, g(x)\rangle+h(\bar{e})-\langle y, \bar{e}\rangle \geq V(0)-D \quad \forall(x, \bar{e}) \in \mathbb{X} \times \mathbb{E} & \Leftrightarrow \\
f(x)+\langle y, g(x)\rangle-h^{*}(y) \geq V(0)-D \quad \forall x \in \mathbb{X} & \Leftrightarrow \\
\inf _{x} L(x, y) \geq \inf (P)-D \Leftrightarrow y \in M_{D} . &
\end{array}
$$

Proposition 3.2 (Weak and strong stability of problem $(P)[4]$ ). The value function $V$ is weakly subdifferentiable at 0 -and problem $(P)$ is then declared weakly stable-iff 
$\inf (P)$ is finite and equals the saddle value

$$
\inf _{x} \sup _{y} L(x, y)=\sup _{y} \inf _{x} L(x, y) .
$$

The same function $V$ is subdifferentiable at 0 - and problem $(P)$ is then called strongly stable-iff $\inf (P)$ is finite and equals the saddle value

$$
\inf _{x} L(x, \bar{y})=\inf _{x} \sup _{y} L(x, y) \text { for each Lagrange multiplier } \bar{y} \text {. }
$$

Proof. By Proposition 3.1

$$
\begin{array}{lll}
\forall D>0 & \partial_{D} V(0) \neq \emptyset & \Leftrightarrow \\
\forall D>0 & \exists y \in M_{D} & \Leftrightarrow \\
\forall D>0 & \exists y \text { such that } \inf _{x} L(x, y) \geq V(0)-D \Leftrightarrow \\
\sup _{y} \inf _{x} L(x, y) \geq V(0)=\inf (P) . &
\end{array}
$$

In this string, any $\bar{y} \in \partial V(0)=M$ invariably applies to yield

$$
\sup _{y} \inf _{x} L(x, y) \geq \inf _{x} L(x, \bar{y}) \geq \inf (P) .
$$

In addition, the inequality $f(x)+h(g(x)) \geq f(x)+\langle y, g(x)\rangle-h^{*}(y)$ is valid for all $(x, y) \in$ $\mathbb{X} \times \mathbb{Y}$. Consequently, $\inf (P) \geq \inf _{x} \sup _{y} L(x, y)$. Thus, the inequality $\inf _{x} L(x, \bar{y}) \geq \inf (P)$ is squeezed in sandwich:

$$
\sup _{y} \inf _{x} L(x, y) \geq \inf _{x} L(x, \bar{y}) \geq \inf (P) \geq \inf _{x} \sup _{y} L(x, y) .
$$

Equalities hold in (9) because $\sup _{y} \inf _{x} L(x, y) \leq \inf _{x} \sup _{y} L(x, y)$.

Corollary 3.2 (Biconjugacy and weak stability). Problem $(P)$ is weakly stable iff the biconjugate of marginal function

$$
V^{* *}(e):=\sup \left\{\langle y, e\rangle-V^{*}(e): y \in \mathbb{Y}\right\}
$$

satisfies $V^{* *}(0)=V(0) \in \mathbb{R}$.

Proof. From $V^{*}(y)=-\inf _{x} L(x, y)$ it follows that $V^{* *}(0)=\sup _{y} \inf _{x} L(x, y)$. If $(P)$ is weakly stable, the last entity equals $\inf (P)=V(0) \in \mathbb{R}$. Conversely, $V^{* *}(0) \geq V(0) \in \mathbb{R}$ amounts to have the left-most inequality in $(9)$. Since the other inequality there came for free, weak stability of $(P)$ follows.

It is common in Lagrangian duality to recover the primal objective $\operatorname{as~}_{\sup _{y}} L(x, y)$. But here it only holds that

$$
\sup _{y} L(x, y)=f(x)+h^{* *}(g(x)) \leq f(x)+h(g(x))
$$

because the biconjugate $h^{* *}$ always satisfies $h^{* *} \leq h$. This observation indicates advantages in selecting $h$ convex, the purpose then being to achieve $h=h^{* *}$. Anyway, if inf $(P)$ is attained at $\bar{x}$, then necessarily $h(g(\bar{x}))=h^{* *}(g(\bar{x}))$, this telling that $h$, in such circumstances, must indeed be "convex" at the point $g(\bar{x})$. Although attainment of $\inf (P)$ is not our chief concern, it's worthwhile to record how Lagrange multipliers relate to primal optimal solutions:

Proposition 3.3 (Lagrange multipliers as subdifferentials of $h[4]$ ). Suppose problem $(P)$ is stable. 
- If $\bar{x}$ minimizes $(P)$, then any Lagrange multiplier $\bar{y}$ must belong to $\partial h(g(\bar{x}))$ and satisfy

$$
f(\bar{x})+\langle\bar{y}, g(\bar{x})\rangle=\min \{f(x)+\langle\bar{y}, g(x)\rangle: x \in \mathbb{X}\} .
$$

- Conversely, if $(\bar{x}, \bar{y}) \in \mathbb{X} \times \mathbb{Y}$ satisfies (10) with $\bar{y} \in \partial h(g(\bar{x})$, then $\bar{x}$ minimizes $(P)$.

Proof. For the first bullet, given any minimizer $\bar{x}$ of $(P)$ pick an arbitrary $\bar{y} \in M=\partial V(0)$. It holds for each $x$ that

$$
f(\bar{x})+h(g(\bar{x}))=\inf (P) \leq L(x, \bar{y})=f(x)+\langle\bar{y}, g(x)\rangle-h^{*}(\bar{y}) .
$$

Insert $x=\bar{x}$ on the right hand side to have $h(g(\bar{x})) \leq\langle\bar{y}, g(\bar{x})\rangle-h^{*}(\bar{y})$ whence

$$
h(g(\bar{x}))+h^{*}(\bar{y})=\langle\bar{y}, g(\bar{x})\rangle .
$$

This implies first, $\bar{y} \in \partial h(g(\bar{x}))$ and second, (10). For the last bullet, (10) and $\bar{y} \in$ $h(g(\bar{x}) \Leftrightarrow(11)$ yield

$$
f(\bar{x})+h(g(\bar{x}))=\min _{x}\left\{f(x)+\langle\bar{y}, g(x)\rangle-h^{*}(\bar{y})\right\}=\min _{x} L(x, \bar{y}) .
$$

This tells, in view of (9), that $\bar{x}$ minimizes $(P)$-and that $\bar{y} \in M$.

So far, using only algebra and numerical ordering, we have identified deficit multipliers - or equivalently, deficit subgradients - as expedient objects for Lagrangian duality. Needed next are arguments showing that such objects do indeed exist in common circumstances. Recall that a point $c$ in a subset $C$ of real vector space is declared absorbing if for all directions $d \neq 0$ there exists a real $r>0$ such that $c+] 0, r[d \subset C$. As customary epi $V:=\{(e, r) \in \mathbb{E} \times \mathbb{R}: V(e) \leq r\}$ denotes the epigraph of $V$. Upon taking the convex hull Conv of the latter set one gets $C o n v(e p i V)=e p i(\operatorname{conv} V)$ where convV denotes the largest convex function $\leq V$.

Proposition 3.4 (Linear support of $V$ at 0). Suppose 0 is absorbing in domV. Also suppose Conv (epiV) contains an absorbing point, but $(0, V(0))$ is not of that sort. Then, letting $\mathbb{Y}$ consist of all linear $y: \mathbb{E} \rightarrow \mathbb{R}$, the subdifferential $\partial V(0)$ is nonempty.

Proof. By the Hahn-Banach separation theorem there is a hyperplane that supports $C:=\operatorname{Conv}(e p i V)$ in the non-absorbing point $(0, V(0))$. That hyperplane is defined in terms of a linear functional $\left(e^{*}, r^{*}\right) \neq 0$, and

$$
\left\langle e^{*}, e\right\rangle+r^{*} r \geq r^{*} V(0) \text { for all }(e, r) \in C \text {. }
$$

Plainly, $(e, r) \in C \& \bar{r}>r \Rightarrow(e, \bar{r}) \in C$. Consequently, $r^{*} \geq 0$. If $r^{*}=0$, then, since 0 is absorbing in $\operatorname{dom} V$, it holds that $\left\langle e^{*}, e\right\rangle \geq 0$ for all $e \in \mathbb{E}$, whence $e^{*}=0$, and the contradiction $\left(e^{*}, r^{*}\right)=0$ obtains. So, divide through (12) with $r^{*}>0$, define $y:=-e^{*} / r^{*}$, and put $r=V(e)$ to have

$$
V(e) \geq V(0)+\langle y, e\rangle \text { for all } e \in \mathbb{E} \text {. }
$$

That is, $y \in \partial V(0)$.

Proposition 3.5 (Continuous linear support of $V$ at 0 ). Let $\mathbb{E}$ be a topological, locally convex, separated, real vector space. Denote by convV the largest convex function $\leq V$. Suppose $V$ is finite-valued, bounded above on a neighborhood of 0 and conv $V(0)=V(0)$. Then, letting $\mathbb{Y}$ consist of all continuous linear functionals $y: \mathbb{E} \rightarrow \mathbb{R}$, the subdifferential $\partial V(0)$ is nonempty. 
Propositions 3.4-5 emphasize the convenience of $(0, V(0))$ being "non-interior" to Conv $(e p i V)$. In particular, it simplifies things to have epi $\mathrm{C}$ or equivalently, $V$ itselfconvex.

4. Core deficit and duality gap. After this detour its is time to reconsider production games with coalition costs $C_{S}$ defined in $\left(P_{S}\right)$. As indicated, we seek explicit cost allocations $c=\left(c_{i}\right) \in D$-core with small deficit $D$. For that purpose, in view of Example 2.1 and Proposition 2.1, recall that

$$
L_{S}\left(x_{S}, y\right)=f_{S}\left(x_{S}\right)+\left\langle y, g_{S}\left(x_{S}\right)\right\rangle-h_{S}^{*}(y)
$$

is the Lagrangian of coalition $S$, and introduce a standing

Hypothesis on AdDitive estimates. Let henceforth $\mathbb{X}_{S}:=\Pi_{i \in S} \mathbb{X}_{i}$ and suppose there exist for each $i$, three functions $F_{i}: \mathbb{X}_{i} \rightarrow \mathbb{R} \cup\{+\infty\}, G_{i}: \mathbb{X}_{i} \rightarrow \mathbb{E} ;$ and $H_{i}: \mathbb{E} \rightarrow \mathbb{R} \cup\{+\infty\}$ such that for all nonempty $S \subseteq I$ and $y \in \mathbb{Y}$,

$$
\inf _{x_{S}} L_{S}\left(x_{S}, y\right) \geq \inf \left\{\sum_{i \in S}\left[F_{i}\left(x_{i}\right)+\left\langle y, G_{i}\left(x_{i}\right)\right\rangle-H_{i}^{*}(y)\right]: x_{i} \in \mathbb{X}_{i}\right\} .
$$

Further, for the grand coalition $S=I$ it should hold that

$$
\inf _{x_{I}} L_{I}\left(x_{I}, y\right) \leq \sup _{y} \inf \left\{\sum_{i \in I}\left[F_{i}\left(x_{i}\right)+\left\langle y, G_{i}\left(x_{i}\right)\right\rangle-H_{i}^{*}(y)\right]: x_{i} \in \mathbb{X}_{i}\right\} .
$$

Proposition 4.1. The standing hypothesis holds if for all nonempty $S \subseteq I, x_{S} \in \mathbb{X}_{S}$, $y \in \mathbb{Y}$

$$
f_{S}\left(x_{S}\right)+\left\langle y, g_{S}\left(x_{S}\right)\right\rangle \geq \sum_{i \in S}\left\{F_{i}\left(x_{i}\right)+\left\langle y, G_{i}\left(x_{i}\right)\right\rangle\right\},
$$

and for all $e \in \mathbb{E}$,

$$
h_{S}(e) \geq \inf \left\{\sum_{i \in S} H_{i}\left(e_{i}\right): \sum_{i \in S} e_{i}=e\right\},
$$

with equalities when $S=I$.

EXAmple 4.1 (Positive homogeneous penalty). Let $h: \mathbb{E} \rightarrow \mathbb{R} \cup\{+\infty\}$ be positively homogeneous. For example, $h$ could be the support function of some nonempty subset of a vector space that is pre-dual to $\mathbb{E}$. Then $h^{*}$, restricted to $\mathbb{Y}$, is the extended indicator $\delta_{Y}$ of some convex set $Y \subseteq \mathbb{Y}$. That is, $h^{*}(y)=0$ if $y \in Y,+\infty$ otherwise. Suppose $h^{*}=H_{i}^{*}=h_{S}^{*}$ for all $i \in I$ and all $S \subseteq I$. Then (14) implies (13).

EXAMPle 4.2 (Cone constraints). Of special notice is the instance when $h$ equals the extended indicator $\delta_{K}$ of a convex cone $K \subset \mathbb{E}$. Then $h^{*}=\delta_{K^{*}}$ where $K^{*}:=\{y:\langle y, K\rangle$ $\leq 0\}$ is the negative dual (polar) cone. In Example 2.1 let all $K_{i}$ be the same convex cone $K \subset \mathbb{E}$ and posit $H_{i}^{*}=h_{S}^{*}:=h^{*}$ for all $i \in I$ and all $S \subseteq I$. Then the above hypothesis is satisfied, and coalition $S$ incurs stand-alone cost

$$
C_{S}:=\inf \left\{\sum_{i \in S} f_{i}\left(x_{i}\right): \sum_{i \in S} g_{i}\left(x_{i}\right) \in K, x_{i} \in \mathbb{X}_{i}\right\} .
$$

Observe that costs and constraints are here pooled additively. However, no activity set can be transferred from any agent to another. 
EXAMPLE 4.3 (Inf-convolution of penalties). When

$$
h_{S}(e):=\inf \left\{\sum_{i \in S} h_{i}\left(x_{i}\right): \sum_{i \in S} x_{i}=e\right\},
$$

we get $h_{S}^{*}(y)=\sum_{i \in S} h_{i}^{*}(y)$ and may thus use $H_{i}^{*}=h_{i}^{*}$.

Proposition 4.2 (Explicit allocations in D-core). Each D-multiplier for problem $\left(P_{I}\right)$ -that is, for the grand coalition $S=I$-generates a cost allocation $c=\left(c_{i}\right) \in D$-core by the formula

$$
c_{i}=c_{i}(y):=\inf \left\{F_{i}\left(x_{i}\right)+\left\langle y, G_{i}\left(x_{i}\right)\right\rangle-H_{i}^{*}(y): x_{i} \in \mathbb{X}_{i}\right\} .
$$

Proof. By the standing hypothesis it holds for any "price regime" $y \in \mathbb{Y}$ and each coalition $S$ that

$$
\begin{aligned}
\sum_{i \in S} c_{i}(y) & \leq \inf _{x_{S}} L_{S}\left(x_{S}, y\right) \leq \sup _{y} \inf _{x_{S}} L_{S}\left(x_{S}, y\right) \leq \inf _{x_{S}} \sup _{y} L_{S}\left(x_{S}, y\right) \\
& =\inf _{x_{S}}\left\{f_{S}\left(x_{S}\right)+h_{S}^{* *}\left(g_{S}\left(x_{S}\right)\right)\right\} \leq \inf _{x_{S}}\left\{f_{S}\left(x_{S}\right)+h_{S}\left(g_{S}\left(x_{S}\right)\right)\right\}=C_{S} .
\end{aligned}
$$

So, no coalition ought reasonably block a proposed payment scheme of the sort $i \mapsto c_{i}(y)$. In addition, $y \in M_{D}\left(P_{I}\right)$ entails

$$
\sum_{i \in I} c_{i}(y)=\inf _{x_{I}} L_{I}\left(x_{I}, y\right) \geq C_{I}-D
$$

Proposition 4.3 (Overestimating the core-deficit). The duality gap

$$
\delta:=\inf _{x_{I}} \sup _{y} L_{I}\left(x_{I}, y\right)-\sup _{y} \inf _{x_{I}} L_{I}\left(x_{I}, y\right)
$$

majorizes the minimal core-deficit; that is: $\delta \geq d \geq 0$. In particular, if $\delta=0$, then core $\neq \emptyset$.

Proof. By assumption $C_{I}=\inf \left(P_{I}\right)$ is finite. For each deficit $D>\delta$ there is a $D$ multiplier $y$. By Proposition 4.1 this $y$ generates a $c \in D$-core. Consequently, $\delta \geq d$.

THEOREM 4.1 (Weak and strong stability of $\left(P_{I}\right)$ yield nonempty core). If problem $\left(P_{I}\right)$ is weakly stable; that is, if $V(0)=V^{* *}(0)$-or equivalently, if

$$
\sup _{y} \inf _{x_{I}} L_{I}\left(x_{I}, y\right)=\inf _{x_{I}} \sup _{y} L_{I}\left(x_{I}, y\right),
$$

then core is nonempty. If moreover, $\left(P_{I}\right)$ stable, then each of its Lagrange multipliers $y$ defines a cost allocation (15) that belongs to core.

EXAMPLE 4.4 (Cooperative linear programming). A special and important version of Example 2.1 has $\mathbb{X}_{i}:=\mathbb{R}_{+}^{n_{i}}$, cost function $k_{i}^{T} x_{i}$ with $k_{i} \in \mathbb{R}^{n_{i}}, g_{i}\left(x_{i}\right):=A_{i} x_{i}-e_{i}$ with $e_{i} \in \mathbb{R}^{m}$ and $A_{i}$ a $m \times n_{i}$ matrix. Posit $K_{i}:=\{0\}$ for all $i$ to get, for coalition $S$, a stand-alone cost given by the standard linear program

$$
C_{S}:=\inf \left\{\sum_{i \in S} k_{i}^{T} x_{i}: \sum_{i \in S} A_{i} x_{i}=\sum_{i \in S} e_{i} \text { with } x_{i} \geq 0 \text { for all } i\right\}
$$

$\left(\right.$ linear $\left.P_{S}\right)$

Suppose primal problem (linear $\left.P_{I}\right)$ and its dual

$$
\sup \left\{y^{T} \sum_{i \in I} e_{i}: A_{i}^{T} y \leq k_{i} \text { for all } i\right\}
$$

$\left(\right.$ linear $\left.D_{I}\right)$

are both feasible. Then $\inf \left(\right.$ linear $\left.D_{I}\right)$ is attained and, by Theorem 4.1, for any dual 
optimal solution $\bar{y}$ the payment scheme $c_{i}:=\bar{y}^{T} e_{i}$ yields $\left(c_{i}\right) \in$ core. Thus, regarding $e_{i}$ as the production target of "factory" or corporate division $i$, those targets are evaluated by a common price $\bar{y}$. Extensions to linear games involving general cones is immediate [1].

EXAMPLE 4.5 (Inf-convolution continued). Each Lagrange multiplier $\bar{y}$ that applies to Example 2.1, generates a cost allocation $\left(c_{i}\right) \in$ core via

$$
c_{i}:=\left\langle\bar{y}, e_{i}\right\rangle-f_{i}^{*}(\bar{y})
$$

This formula is quite telling: agent $i$ is charged $\left\langle\bar{y}, e_{i}\right\rangle$ for his production task less the price-taking profit $f_{i}^{*}(\bar{y})$ he can generate, both entities calculated at shadow prices.

I end this section by overestimating the deficit $d$ when $\mathbb{E}$ is finite-dimensional.

Definition (Vertical convexity deficit). For any subset $\mathcal{E} \subset \mathbb{E} \times \mathbb{R}$ its vertical convexity deficit is defined by

$$
v \operatorname{def}(\mathcal{E}):=\inf \{r>0: \operatorname{Conv} \mathcal{E} \subseteq \mathcal{E}+\{0\} \times[-r, r]\}
$$

The common convention $\inf \emptyset=+\infty$ applies here. Clearly, if $\mathcal{E} \subset \mathbb{E} \times \mathbb{R}$ is convex, then $\operatorname{vde} f(\mathcal{E})=0$. Also, for any finite family of subsets $\mathcal{E}_{i}, i \in I$, it holds that $\operatorname{vde} f\left(\sum_{i \in I} \mathcal{E}_{i}\right) \leq$ $\sum_{i \in I} v \operatorname{def}\left(\mathcal{E}_{i}\right)$. Indeed, when $r_{i}>\operatorname{vdef}\left(\mathcal{E}_{i}\right)$ for all $i$, and $r:=\sum_{i \in I} r_{i}$

$$
\text { Conv } \sum_{i \in I} \mathcal{E}_{i} \subseteq \sum_{i \in I} \operatorname{Conv} \mathcal{E}_{i} \subseteq \sum_{i \in I}\left\{\mathcal{E}_{i}+\{0\} \times\left[-r_{i}, r_{i}\right]\right\} \subseteq \sum_{i \in I} \mathcal{E}_{i}+\{0\} \times[-r, r] .
$$

Proposition 4.4 (Overestimating the vertical deficit). Let $\mathbb{E}$ be finite-dimensional. For any finite family $\mathcal{E}_{i}, i \in I$, of nonempty subsets in $\mathbb{E} \times \mathbb{R}$ it holds that

$$
\operatorname{vdef}\left(\sum_{i \in I} \mathcal{E}_{i}\right) \leq(\operatorname{dim} \mathbb{E}+2) \max _{i \in I} \operatorname{vdef}\left(\mathcal{E}_{i}\right) \text {. }
$$

Proof. The Folkman-Shapley Lemma says that any $x \in \operatorname{Conv} \sum_{i \in I} \mathcal{E}_{i}$ can be synthesized as a sum $x=\sum_{i \in I} x_{i}$ with $x_{i} \in \operatorname{Conv}_{i}$ for all $i$ in some subset $S \subseteq I$, with cardinality $|S| \leq \operatorname{dim} \mathbb{E}+2$, and $x_{i} \in \mathcal{E}_{i}$ otherwise. Select any $r_{i}>\operatorname{vdef}\left(\mathcal{E}_{i}\right)$ and posit $r:=\sum_{i \in S} r_{i}$. Then

$$
x \in \sum_{i \in S} \operatorname{Conv}_{i}+\sum_{i \notin S} \mathcal{E}_{i} \subseteq \sum_{i \in S}\left\{\mathcal{E}_{i}+\{0\} \times\left[-r_{i}, r_{i}\right]\right\}+\sum_{i \notin S} \mathcal{E}_{i} \subseteq \sum_{i \in I} \mathcal{E}_{i}+\{0\} \times[-r, r] .
$$

and the conclusion follows.

THEOREM 4.2 (Overestimating the core deficit) Let $\mathbb{E}$ be finite-dimensional. A production game featuring $f_{S}\left(x_{S}\right):=\sum_{i \in S} f_{i}\left(x_{i}\right), g_{S}\left(x_{S}\right):=\sum_{i \in S} g_{i}\left(x_{i}\right)$, and

$$
h_{S}(e)=\inf \left\{\sum_{i \in S} h_{i}\left(e_{i}\right): \sum_{i \in S} e_{i}=e\right\} \forall S \subseteq I,
$$

with each $h_{i}$ lower semicontinuous convex, has minimal core deficit

$$
d \leq(\operatorname{dim} \mathbb{E}+2) \max _{i \in I} v \operatorname{def}\left(\mathcal{E}_{i}\right)
$$

where

$$
\mathcal{E}_{i}:=\left\{(e, r) \in \mathbb{E} \times \mathbb{R}: f_{i}(x) \leq r \text { and } g_{i}(x)=e \text { for some }(x, r) \in \mathbb{X}_{i} \times \mathbb{R}\right\} .
$$

Proof. Introduce the "partial sign shift" $\pi(e, r):=(-e, r)$ and the "strict" set

$$
\mathcal{E}_{i}^{+}:=\left\{(e, r) \in \mathbb{E} \times \mathbb{R}: f_{i}(x)<r \text { and } g_{i}(x)=e \text { for some } x \in \mathbb{X}_{i}\right\} .
$$


It follows from [4] that

$$
e p i^{+} V_{I}=\pi \circ \sum_{i \in I} \mathcal{E}_{i}^{+}+\sum_{i \in I} e p i^{+} h_{i}
$$

where $e p i^{+} h_{i}:=\left\{(e, r) \in \mathbb{E} \times \mathbb{R}: h_{i}(e)<r\right\}$ is the strict epigraph of $h_{i}$. Clearly, $\operatorname{vdef}\left(\operatorname{epih}_{i}^{+}\right)=0$, and $\operatorname{vdef}\left(\pi \circ \sum_{i \in I} \mathcal{E}_{i}^{+}\right)=\operatorname{vdef} \sum_{i \in I} \mathcal{E}_{i}$. Since $d \leq \operatorname{vdef}\left(e p i^{+} V_{I}\right)$, the conclusion follows from Proposition 4.4.

5. Conclusion. Broadly, the paper shows that provided costs and technologies be additively separable, to have an explicit, computable, core solution of the production/market game one really need not convexity all over the place. It suffices that the aggregated marginal function $V_{I}$ be subdifferentiable (in the sense of convex analysis) at the point $e=0$ of no perturbation.

\section{References}

[1] A. Ben-Tal and A. Nemirovski, Lectures on Modern Convex Optimization, SIAM, Philadelphia (2001).

[2] O. N. Bondareva, Some apllications of linear programming methods to the theory of cooperative games, Problemy Kibernetiki 10 (1963), 119-139.

[3] E. Bennet, The aspiration approach to predicting coalition formation and payoff distribution in sidepayment games, Int. J. Game Theory 12 (1983), 1-28.

[4] A. Bourass and E. Giner, Kuhn-Tucker conditions and integral functionals, J. Convex Analysis 8 (2001), 533-553.

[5] P. Dubey and L. S. Shapley, Totally balanced games arising from controlled programming problems, Mathematical Programing 29 (1984), 245-276.

[6] I. V. Evstigneev and S. D. Flåm, Sharing nonconvex cost, J. Global Optimization 20 (2001), 257-271.

[7] J. C. Gomez, An extension of the core solution concept, Discussion Paper 04-01, Institute of Economics, University of Copenhagen, 2004.

[8] J. C. Gomez, Axiomatizing a nonempty core extension, Discussion Paper, Institute of Economics, University of Copenhagen, 2004.

[9] D. Granot, A generalized linear production model: a unifying model, Mathematical Programming 43 (1986), 212-222.

[10] E. Kalai and E. Zemel, Generalized network problems yielding totally balanced games, Operations Research 30 (1982), 998-1008.

[11] H. Keiding, An axiomatization of the aspiration core, manuscript, 2004.

[12] R. B. Myerson, Game Theory, Harvard University Press, Cambridge, 1991.

[13] D. Samet and Zemel, On the core and dual set of linear programming games, Mathematics of Operations Research 9 (1994), 309-316.

[14] M. Sandsmark, Production games under uncertainty, Computational Economics (1999).

[15] L. S. Shapley, On balanced sets and cores, Naval Research Logistics Quarterly 14 (1967), 453-461.

[16] L. S. Shapley and M. Shubik, On market games, J. Economic Theory 1 (1969), 9-25.

[17] G. Owen, On the core of linear production games, Mathematical Programming 9 (1975), $358-370$. 